\title{
Graph-Based Ontology Classification in OWL 2 QL
}

\author{
Domenico Lembo, Valerio Santarelli, and Domenico Fabio Savo \\ Dipartimento di Ing. Informatica, Automatica e Gestionale "Antonio Ruberti" \\ SAPIENZA Università di Roma \\ Via Ariosto 25, I-00186 Roma, Italy \\ \{lembo, santarelli, savo\}@dis . uniroma1.it
}

\begin{abstract}
Ontology classification is the reasoning service that computes all subsumption relationships inferred in an ontology between concept, role, and attribute names in the ontology signature. OWL 2 QL is a tractable profile of OWL 2 for which ontology classification is polynomial in the size of the ontology TBox. However, to date, no efficient methods and implementations specifically tailored to OWL 2 QL ontologies have been developed. In this paper, we provide a new algorithm for ontology classification in OWL 2 QL, which is based on the idea of encoding the ontology TBox into a directed graph and reducing core reasoning to computation of the transitive closure of the graph. We have implemented the algorithm in the QUONTO reasoner and extensively evaluated it over very large ontologies. Our experiments show that QUONTO outperforms various popular reasoners in classification of OWL 2 QL ontologies.
\end{abstract}

\section{Introduction}

Ontology classification is the problem of computing all subsumption relationships inferred in an ontology between predicate names in the ontology signature, i.e., named concepts (a.k.a. classes), roles (a.k.a. object-properties), and attributes (a.k.a. data-properties). It is considered a core service for ontology reasoning, which can be exploited for various tasks, at both design-time and run-time, ranging from ontology navigation and visualization to query answering.

Devising efficient ontology classification methods and implementations is a challenging issue, since classification is in general a costly operation. Most popular reasoners for Description Logic (DL) ontologies, i.e., OWL ontologies, such as Pellet 22, Racer [11, FACT ++ [23, and HermiT [9], offer highly optimized classification services for expressive DLs. Various experimental studies show that such reasoners have reached very good performances through the years. However, they are still not able to efficiently classify very large ontologies, such as the full versions of GALEN [21] or of the FMA ontology [10].

Whereas the above tools use algorithms based on model construction through

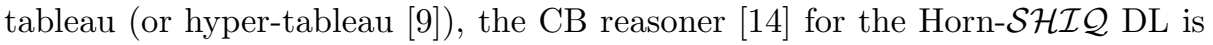
a consequence-driven reasoner. The use of this technique allows CB to obtain an impressive gain on very large ontologies, such as full GALEN. However, the

P. Cimiano et al. (Eds.): ESWC 2013, LNCS 7882, pp. 320-334, 2013.

(C) Springer-Verlag Berlin Heidelberg 2013 
current implementation of the $\mathrm{CB}$ reasoner is rather specific for particular fragments of Horn- $\mathcal{S H \mathcal { I }}$ (and incomplete for the general case) 14. For example, it does not allow for classification of properties.

Other recently developed tools, such as Snorocket [17], ELK [15], and JCEL [19, are specifically tailored to intensional reasoning over logics of the $\mathcal{E} \mathcal{L}$ family, and show excellent performances in classification of ontologies specified in such languages, which are the logical underpinning of OWL 2 EL, one of the tractable profile of OWL 2 [20].

Instead, to the best of our knowledge, ontology classification in the other OWL 2 profiles has received so far little attention. In particular, classification in OWL 2 RL has been investigated only in [16, whereas, to date, no techniques have been developed that are specifically tailored to intensional reasoning in OWL 2 QL, the "data oriented" profile of OWL 2, nor for any logic of the DL-Lite family [6, which constitutes the logical underpinning of OWL 2 QL. Our aim is then to contribute to fill this lack on OWL $2 \mathrm{QL}$, encouraged also by the fact that such language, like all logics of the $D L$-Lite family, allows for tractable intensional reasoning, and in particular for PTIME ontology classification, as it immediately follows from the results in [6].

In this paper, we thus provide a new method for ontology classification in the OWL 2 QL profile. In our technique, we encode the ontology terminology (TBox) into a graph, and compute the transitive closure of the graph to then obtain the ontology classification. The analogy between simple inference rules in DLs and graph reachability is indeed very natural: consider, for example, an ontology containing the subsumptions $A_{1} \sqsubseteq A_{2}$ and $A_{2} \sqsubseteq A_{3}$, where $A_{1}, A_{2}$, and $A_{3}$ are class names in the ontology signature. We can then associate to this ontology a graph having three nodes labeled with $A_{1}, A_{2}$, and $A_{3}$, respectively, an edge from $A_{1}$ to $A_{2}$ and an edge from $A_{2}$ to $A_{3}$. It is straightforward to see that $A_{3}$ is reachable from $A_{1}$, and therefore an edge from $A_{1}$ to $A_{3}$ is contained in the transitive closure of the graph. This corresponds to the inferred subsumption $A_{1} \sqsubseteq A_{3}$. On the other hand, things become soon much more complicated when complex (OWL) axioms come into play.

In this respect, we will show that for an OWL 2 QL ontology it is possible to easily construct a graph whose closure constitutes the major sub-task in ontology classification, because it allows us to obtain all subsumptions inferred by the "positive knowledge" specified by the TBox. We will show that the computed classification misses only "trivial" subsumptions inferred by unsatisfiable predicates, i.e., named classes (resp. properties) that always have an empty interpretation in every model of the ontology, and that are therefore subsumed by every class (resp. property) in the ontology signature. We therefore provide an algorithm that, exploiting the transitive closure of the graph, computes all unsatisfiable predicates, thus allowing us to obtain a complete ontology classification. We notice that the presence of unsatisfiable predicates in an ontology is mainly due to errors in the design. However, it is not rare to find such predicates, especially in very large ontologies or in ontologies that are still "under construction". In particular, we could find unsatisfiable concepts even in some 
benchmark ontologies we used in our experiments (cf. Section 4). Of course, already debugged ontologies might not present such predicates [13]12. In this case, one can avoid executing our algorithm for computing unsatisfiable predicates.

We have implemented our technique in a new module of QuOnTo [1, the reasoner at the base of the MASTRO [517] system, and have carried out extensive experimentation, focusing in particular on very large ontologies. We have considered well-known ontologies, often used as benchmark for ontology classification, and we have suitably approximated them in OWL 2 QL.

QUONTO showed better performances, in some cases corresponding to enormous gains, with respect to tableau-based reasoners (in particular, Pellet, Fact++, and HermiT). We also obtained comparable or better results with respect to the $\mathrm{CB}$ reasoner, for almost all ontologies considered, but, differently from $\mathrm{CB}$ reasoner, we were always able to compute a complete classification. We finally compared QUONTO with ELK, one of the most performing reasoner for $\mathcal{E} \mathcal{L}$, for those approximated ontologies that turned out to be both in OWL 2 QL and OWL 2 EL, obtaining similar performances in almost all cases.

We conclude by noticing that, even though we refer here to OWL 2 QL, our algorithms and implementations can be easily adapted to deal with all logics of the $D L$-Lite family mentioned in [6, excluding those allowing for the use of conjunction in the left-hand side of inclusion assertions or the use of $n$-ary relations instead of binary roles.

The rest of the paper is organized as follows. In Section 2, we provide some preliminaries. In Section 3 , we describe our technique for ontology classification in OWL 2 QL. In Section 4 we describe our experimentation, and finally, in Section [5] we conclude the paper.

\section{Preliminaries}

In this section, we present some basic notions on DL ontologies, the formal underpinning of the OWL 2 language, and on OWL 2 QL. We also recall some notions of graph theory needed later on.

Description Logic Ontologies. We consider a signature $\Sigma$, partitioned in two disjoint signatures, namely, $\Sigma_{P}$, containing symbols for predicates, i.e., atomic concepts, atomic roles, atomic attributes, and value-domains, and $\Sigma_{C}$, containing symbols for individual (object and value) constants. Complex concept, role, and attribute expressions are constructed starting from predicates of $\Sigma_{P}$ by applying suitable constructs, which vary in different DL languages. Given a DL language $\mathcal{L}$, an $\mathcal{L}$-TBox (or simply a TBox, when $\mathcal{L}$ is clear) over $\Sigma$ contains universally quantified first-order (FOL) assertions, i.e., axioms specifying general properties of concepts, roles, and attributes. Again, different DLs allow for different axioms. An $\mathcal{L}$-ABox (or simply an ABox, when $\mathcal{L}$ is clear) is a set of assertions on individual constants, which specify extensional knowledge. An $\mathcal{L}$-ontology $\mathcal{O}$ is constituted by both an $\mathcal{L}$-TBox $\mathcal{T}$ and an $\mathcal{L}$-ABox $\mathcal{A}$, denoted as $\mathcal{O}=\langle\mathcal{T}, \mathcal{A}\rangle$.

The semantics of a DL ontology $\mathcal{O}$ is given in terms of FOL interpretations (cf. [3]). We denote with $\operatorname{Mod}(\mathcal{O})$ the set of models of $\mathcal{O}$, i.e., the set of FOLinterpretations that satisfy all TBox axioms and ABox assertions in $\mathcal{O}$, where 
the definition of satisfaction depends on the DL language in which $\mathcal{O}$ is specified. An ontology $\mathcal{O}$ is satisfiable if $\operatorname{Mod}(\mathcal{O}) \neq \emptyset$. A FOL-sentence $\phi$ is entailed by an ontology $\mathcal{O}$, denoted $\mathcal{O}=\phi$, if $\phi$ is satisfied by every model in $\operatorname{Mod}(\mathcal{O})$. All the above notions naturally apply to a TBox $\mathcal{T}$ alone.

Traditional intensional reasoning tasks with respect to a given TBox are verification of subsumption and satisfiability of concepts, roles, and attributes [3]. More precisely, a concept $C_{1}$ is subsumed in $\mathcal{T}$ by a concept $C_{2}$, written $\mathcal{T} \models C_{1} \sqsubseteq C_{2}$, if, in every model $I$ of $\mathcal{T}$, the interpretation of $C_{1}$, denoted $C_{1}^{I}$, is contained in the interpretation of $C_{2}$, denoted $C_{2}^{I}$, i.e., $C_{1}^{I} \subseteq C_{2}^{I}$ for every $I \in \operatorname{Mod}(\mathcal{T})$. Furthermore, a concept $C$ in $\mathcal{T}$ is unsatisfiable, which we wrote as $\mathcal{T} \models C \sqsubseteq \neg C$, if the interpretation of $C$ is empty in every model of $\mathcal{T}$, i.e., $C^{I}=\emptyset$ for every $I \in \operatorname{Mod}(\mathcal{T})$. Analogous definitions hold for roles and attributes.

Strictly related to the previous reasoning tasks is the classification inference service, which we focus on in this paper. Given a signature $\Sigma_{P}$ and a TBox $\mathcal{T}$ over $\Sigma_{P}$, such a service allows to determine subsumption relationships in $\mathcal{T}$ between concepts, roles, and attributes in $\Sigma_{P}$. Therefore, classification allows to structure the terminology of $\mathcal{T}$ in the form of a subsumption hierarchy that provides useful information on the connection between different terms, and can be used to speed up other inference services. Here we define it more formally.

Definition 1. Let $\mathcal{T}$ be a satisfiable $\mathcal{L}$-TBox over $\Sigma_{P}$. We define the $\mathcal{T}$ classification of $\Sigma_{P}$ (or simply $\mathcal{T}$-classification when $\Sigma_{P}$ is clear from the context) as the set of inclusion assertions defined as follows:

Let $S_{1}$ and $S_{2}$ be either two concepts, roles, or attributes in $\Sigma_{P}$. If

$\mathcal{T} \models S_{1} \sqsubseteq S_{2}$ then $S_{1} \sqsubseteq S_{2}$ belongs to the $\mathcal{T}$-classification of $\Sigma_{P}$.

The OWL 2 QL Language. We now present OWL 2 QL. We use the German notation for describing its constructs and axioms, and refer the reader to [20] for the OWL functional style syntax.

Expressions in OWL 2 QL are formed according to the following syntax:

$$
\begin{array}{l|l|l|l|lll|l}
B \longrightarrow A & \exists Q & \delta(U) & R \longrightarrow Q & \neg Q & E \longrightarrow \rho(U) & \\
C \longrightarrow B & \neg B & \exists Q . A \mid \delta_{F}(U) & V \longrightarrow U & \neg U & F \longrightarrow T_{1} \mid & \ldots & T_{n} \\
Q \longrightarrow P & P^{-} & & & &
\end{array}
$$

where: $A, P$, and $U$ are symbols in $\Sigma_{P}$ denoting respectively an atomic concept, an atomic role, and an atomic attribute; $P^{-}$denotes the inverse of $P ; \exists Q$, also called unqualified existential role, denotes the set of objects related to some object by the role $Q ; \delta(U)$ denotes the domain of $U$, i.e., the set of objects that $U$ relates to values; $\rho(U)$ denotes the range of $U$, i.e., the set of values related to objects by $U ; T_{1}, \ldots, T_{n}$ denote $n$ unbounded value-domains (i.e., datatypes); the concept $\exists Q$.A, or qualified existential role, denotes the qualified domain of $Q$ with respect to $A$, i.e., the set of objects that $Q$ relates to some instance of $A$. Similarly, $\delta_{F}(U)$ denotes the qualified domain of $U$ with respect to a valuedomain $F$, i.e., the set of objects that $U$ relates to some value in $F$. In the following, we call $B$ a basic concept, and $Q$ a basic role. 
An OWL 2 QL TBox $\mathcal{T}$ is a finite set of axioms of the form:

$$
B \sqsubseteq C \quad Q \sqsubseteq R \quad U \sqsubseteq V \quad E \sqsubseteq F
$$

From left to right, the above axioms denote subsumptions between concepts, roles, attributes, and value-domains, respectively. We call positive inclusions axioms of the form $B_{1} \sqsubseteq B_{2}, B_{1} \sqsubseteq \exists Q . A, B_{1} \sqsubseteq \delta_{F}(U), Q_{1} \sqsubseteq Q_{2}$, and $U_{1} \sqsubseteq U_{2}$, value-domain inclusions axioms of the form $E \sqsubseteq F$, and negative inclusions axioms of the form $B_{1} \sqsubseteq \neg B_{2}, Q_{1} \sqsubseteq \neg Q_{2}$ and $U_{1} \sqsubseteq \neg U_{2}$.

We notice that also other constructs and axioms are in fact allowed in OWL 2 QL. In particular, it allows for the use of $\delta_{F}(U)$ in the left-hand side of subsumptions, or in the right-hand side of negative inclusions, the use of "top" constructs in the left hand-side of subsumptions, corresponding to rdfs:Literal, owl:Thing, owl:topObjectProperty, and owl:topDataProperty, and the use of reflexivity and irreflexivity on roles (i.e., object-properties). For the sake of presentation, in this paper we prefer to not consider such aspects of OWL 2 QL, since their presence requires to burden our algorithms with some technicalities, which represent very minor contributions of our approach. Also, such constructs and axioms are rarely used in the practice, and in particular are never used in the benchmark ontologies considered in our experimentations (cf. Section 4). We notice however, that all the techniques presented in the following sections can be extended to full OWL 2 QL with minimal adaptations. Other constructs, such us symmetric or asymmetric roles, even though not explicitly mentioned, can be easily expressed by the OWL 2 QL syntax we consider.

As for OWL 2 QL ABoxes, we do not present them here, since we concentrate on intensional reasoning, and refer the interested reader to 20].

The semantics of OWL 2 QL ontologies and TBoxes is given in the standard way 20 3. We only recall here that, datatypes, i.e., value-domains, have a fixed predefined interpretation, i.e., each datatype $T_{i}$ is interpreted always in the same way, denoted $\operatorname{val}\left(T_{i}\right)$, in every interpretation of the ontology. Notice also that OWL 2 QL supports only OWL datatypes such that the intersection of the value spaces of any set of these datatypes is either infinite or empty, i.e., for each $i, j \in$ $\{1, \ldots, n\}$, it holds either that $\operatorname{val}\left(T_{i}\right) \cap \operatorname{val}\left(T_{j}\right)$ is infinite or $\operatorname{val}\left(T_{i}\right) \cap \operatorname{val}\left(T_{j}\right)=\emptyset$.

Graph Theory Notions. In this paper we use the term digraph to refer to a directed graph. We assume that a digraph $\mathcal{G}$ is a pair $(\mathcal{N}, \mathcal{E})$, where $\mathcal{N}$ is a set of elements called nodes, and $\mathcal{E}$ is a set of ordered pairs $(s, t)$ of nodes in $\mathcal{N}$, called arcs, where $s$ is denoted the source of the arc, and $t$ the target of the arc.

The transitive closure $\mathcal{G}^{*}=\left(\mathcal{N}, \mathcal{E}^{*}\right)$ of a digraph $\mathcal{G}=(\mathcal{N}, \mathcal{E})$ is a digraph such that there is an arc in $\mathcal{E}^{*}$ having a node $s$ as source and a node $t$ as target if and only if there is a path from $s$ to $t$ in $\mathcal{G}$. Let $\mathcal{G}=(\mathcal{N}, \mathcal{E})$ be a digraph, and let $n$ be a node in $\mathcal{N}$. We denote with $\operatorname{predecessors}(n, \mathcal{G})$ the set of nodes $p_{n}$ in $\mathcal{N}$ such that there exists in $\mathcal{E}$ an $\operatorname{arc}\left(p_{n}, n\right)$.

\section{T-Classification in OWL 2 QL}

In this section we describe our approach to computing, given a signature $\Sigma_{P}$ and an OWL 2 QL TBox $\mathcal{T}$ over $\Sigma_{P}$, the $\mathcal{T}$-classification of $\Sigma_{P}$. 
In OWL 2 QL, a subsumption relation between two concepts, roles, or attributes in $\Sigma_{P}$, can be inferred by a TBox $\mathcal{T}$ if and only if $(i) \mathcal{T}$ contains such subsumption; $($ ii $) \mathcal{T}$ contains a set of positive inclusion assertions that together entail the subsumption; or ( $i i i)$, trivially, the subsumed concept, role, or attribute is unsatisfiable in $\mathcal{T}$. The above observation is formalized as follows.

Theorem 1. Let $\mathcal{T}$ be an $O W L 2 Q L$ TBox containing only positive inclusions, and let $S_{1}$ and $S_{2}$ be two atomic concepts, two atomic roles, or two atomic attributes. $S_{1} \sqsubseteq S_{2}$ is entailed by $\mathcal{T}$ if and only if at least one of the following conditions holds:

1. a set $\mathcal{P}$ of positive inclusions exists in $\mathcal{T}$, such that $\mathcal{P} \models S_{1} \sqsubseteq S_{2}$;

2. $\mathcal{T} \models S_{1} \sqsubseteq \neg S_{1}$.

Proof. (sketch) $(\Leftarrow)$ This is trivially proven.

$\left(\Rightarrow\right.$ ) Assume $\mathcal{T} \models S_{1} \sqsubseteq S_{2}$. Towards a contradiction, suppose that both statements 1 and 2 are false. If $\mathcal{T} \models S_{1} \sqsubseteq S_{2}$ then the following cases are conceivable:

(a) $S_{1} \sqsubseteq S_{2} \in \mathcal{T}$, but this implies that statement 1 is true (contradiction);

(b) $S_{1} \sqsubseteq S_{2} \notin \mathcal{T}$ and $S_{1}$ is satisfiable. Since statement 1 does not hold, it remains that there exists a subset $\mathcal{T}^{\prime}$ of $\mathcal{T}$ formed by positive inclusions and at least one negative inclusion such that $\mathcal{T}^{\prime} \models S_{1} \sqsubseteq S_{2}$. It can be shown that in OWL 2 QL negative inclusions do not concur in the entailment of positive inclusions [6], and therefore $S_{1} \sqsubseteq S_{2}$ follows only from the positive inclusions of $\mathcal{T}^{\prime}$, which contradicts that statement 1 is false;

(c) $S_{1} \sqsubseteq S_{2} \notin \mathcal{T}$ and $S_{1}$ is unsatisfiable. But then statement 2 is true (contradiction).

Given a OWL 2 QL TBox $\mathcal{T}$ over a signature $\Sigma_{P}$, we use $\Phi_{\mathcal{T}}$ and $\Omega_{\mathcal{T}}$ to denote two sets of positive inclusions of the form $S_{1} \sqsubseteq S_{2}$, with $S_{1}, S_{2} \in \Sigma_{P}$, such that $\Phi_{\mathcal{T}}$ contains only positive inclusions for which statement 1 holds, and $\Omega_{\mathcal{T}}$ contains only positive inclusions for which statement 2 holds. It is easy to see that $\Phi_{\mathcal{T}}$ and $\Omega_{\mathcal{T}}$ are not disjoint. From Definition 1 and Theorem 1 it follows that the $\mathcal{T}$-classification coincides with the union of the sets $\Phi_{\mathcal{T}}$ and $\Omega_{\mathcal{T}}$.

In the following, we describe our approach to the computation of the $\mathcal{T}$ classification by firstly computing the set $\Phi_{\mathcal{T}}$, and then computing the set $\Omega_{\mathcal{T}}$.

Computation of $\Phi_{\mathcal{T}}$. Given an OWL 2 QL TBox $\mathcal{T}$, in order to compute $\Phi_{\mathcal{T}}$, we encode the set of positive inclusions in $\mathcal{T}$ into a digraph $\mathcal{G}_{\mathcal{T}}$ and compute the transitive closure of $\mathcal{G}_{\mathcal{T}}$ in such a way that each subsumption $S_{1} \sqsubseteq S_{2}$ in $\Phi_{\mathcal{T}}$ corresponds to an arc $\left(S_{1}, S_{2}\right)$ in such transitive closure, and vice versa. The following constructive definition describes the appropriate manner to obtain the digraph TBox representation for our aims.

Definition 2. Let $\mathcal{T}$ be an $O W L 2 Q L$ TBox over a signature $\Sigma_{P}$. We call the digraph representation of $\mathcal{T}$ the digraph $\mathcal{G}_{\mathcal{T}}=(\mathcal{N}, \mathcal{E})$ built as follows:

1. for each atomic concept $A$ in $\Sigma_{P}, \mathcal{N}$ contains the node $A$;

2. for each atomic role $P$ in $\Sigma_{P}, \mathcal{N}$ contains the nodes $P, P^{-}, \exists P, \exists P^{-}$; 
3. for each atomic attribute $U$ in $\Sigma_{P}, \mathcal{N}$ contains the nodes $U$ and $\delta(U)$;

4. for each concept inclusion $B_{1} \sqsubseteq B_{2} \in \mathcal{T}, \mathcal{E}$ contains the arc $\left(B_{1}, B_{2}\right)$;

5. for each role inclusion $Q_{1} \sqsubseteq Q_{2} \in \mathcal{T}, \mathcal{E}$ contains the arcs $\left(Q_{1}, Q_{2}\right)$, $\left(Q_{1}^{-}, Q_{2}^{-}\right),\left(\exists Q_{1}, \exists Q_{2}\right)$, and $\left(\exists Q_{1}^{-}, \exists Q_{2}^{-}\right)$;

6. for each attribute inclusion $U_{1} \sqsubseteq U_{2} \in \mathcal{T}, \mathcal{E}$ contains the arcs $\left(U_{1}, U_{2}\right)$ and $\left(\delta\left(U_{1}\right), \delta\left(U_{2}\right)\right)$;

7. for each concept inclusion $B_{1} \sqsubseteq \exists Q . A \in \mathcal{T}, \mathcal{E}$ contains the arc $\left(B_{1}, \exists Q\right)$;

8. for each concept inclusion $B_{1} \sqsubseteq \delta_{F}(U) \in \mathcal{T}, \mathcal{E}$ contains the arc $\left(B_{1}, \delta(U)\right)$.

The idea is that each node in the digraph $\mathcal{G}_{\mathcal{T}}$ represents a basic concept, a basic role or an attribute, and each arc models a positive inclusion, i.e., a subsumption, contained in $\mathcal{T}$, where the source node of the arc represents the left-hand side of the subsumption and the target node of the arc represents the right-hand side of the subsumption. Observe that for each role inclusion assertion $P_{1} \sqsubseteq P_{2}$ in the TBox $\mathcal{T}$, we also represent as nodes and arcs in the digraph $\mathcal{G}_{\mathcal{T}}$ the entailed positive inclusions $P_{1}^{-} \sqsubseteq P_{2}^{-}, \exists P_{1} \sqsubseteq \exists P_{2}$, and $\exists P_{1}^{-} \sqsubseteq \exists P_{2}^{-}$. We operate in a similar fashion for positive inclusions on attributes in $\mathcal{T}$.

Let $\mathcal{T}$ be an OWL 2 QL TBox and let $\mathcal{G}_{\mathcal{T}}=(\mathcal{N}, \mathcal{E})$ be its digraph representation. We denote with $\mathcal{G}_{\mathcal{T}}^{*}=\left(\mathcal{N}, \mathcal{E}^{*}\right)$ the transitive closure of $\mathcal{G}_{\mathcal{T}}$. Note that by definition of digraph transitive closure, for each node $n \in \mathcal{N}$ there exists in $\mathcal{E}^{*}$ an $\operatorname{arc}(n, n)$. Moreover, in what follows, we denote with $\alpha\left(\mathcal{E}^{*}\right)$ the set of arcs $\left(S_{1}, S_{2}\right) \in \mathcal{E}^{*}$ such that both terms $S_{1}$ and $S_{2}$ denote in $\mathcal{T}$ either two atomic concepts, two atomic roles, or two attributes. Then, the following property holds.

Theorem 2. Let $\mathcal{T}$ be an $O W L 2 Q L$ TBox and let $\mathcal{G}_{\mathcal{T}}=(\mathcal{N}, \mathcal{E})$ be its digraph representation. Let $S_{1}$ and $S_{2}$ be two atomic concepts, two atomic roles, or two atomic attributes. An inclusion assertion $S_{1} \sqsubseteq S_{2}$ belongs to $\Phi_{\mathcal{T}}$ if and only if there exists in $\alpha\left(\mathcal{E}^{*}\right)$ an arc $\left(S_{1}, S_{2}\right)$.

Proof. $($ sketch $)(\Leftarrow)$ This is trivially proven.

$(\Rightarrow)$ To prove the thesis we need to introduce the notion of chase for an OWL 2 QL ontology, which is analogous to the notion of chase given in 6 8 . We first note that every positive inclusion in the TBox can be formulated as a FOL implication of the form

$$
\forall \boldsymbol{x}, \boldsymbol{y} \cdot S(\boldsymbol{x}, \boldsymbol{y}) \rightarrow \exists \boldsymbol{z} \cdot \psi(\boldsymbol{x}, \boldsymbol{z})
$$

where $S$ is an atomic concept, an atomic attribute, or an atomic role, $\psi$ is a single atom or a conjunction of two atoms constructed on predicates of $\Sigma_{P}, \boldsymbol{x}$ is a vector of one or two variables, $\boldsymbol{y}$ and $\boldsymbol{z}$ are vectors of one or zero variables, i.e., they may be missing. For example, a positive inclusion of the form $A_{1} \sqsubseteq A_{2}$ is written as $\forall x \cdot A_{1}(x) \rightarrow A_{2}(x)$, the positive inclusion $\exists P_{1}^{-} \sqsubseteq \exists P_{2}$. $A$ is written as $\forall x, y \cdot P_{1}(x, y) \rightarrow \exists z \cdot P_{2}(y, z) \wedge A(z)$, or the inclusion $U_{1} \sqsubseteq U_{2}$ is written as $\forall x, y \cdot U_{1}(x, y) \rightarrow U_{2}(x, y)$.

Now, let $\mathcal{O}=\langle\mathcal{T}, \mathcal{A}\rangle$ be an OWL 2 QL ontology. Our notion of chase is given inductively as follows. We pose $\operatorname{chase}_{0}(\mathcal{O})=\mathcal{A}$, and for every non-negative integer $i$, we define chase $_{i+1}(\mathcal{O})$ as the set of ABox assertions obtained from chase $_{i}(\mathcal{O})$ by applying the following rule: 
Chase Rule. Let $I$ be a positive inclusion in $\mathcal{T}$ of the form (1). Let $h$ be a homomorphism from $S(\boldsymbol{x}, \boldsymbol{y})$ to $\operatorname{chase}_{i}(\mathcal{O})$ such that $h(S(\boldsymbol{x}, \boldsymbol{y}))=$ $S(\boldsymbol{a}, \boldsymbol{b})$, and such that there is no extension of $h$ to a homomorphism $h^{\prime}$ from $S(\boldsymbol{x}, \boldsymbol{y}) \wedge \psi(\boldsymbol{x}, \boldsymbol{z})$ to $\operatorname{chase}_{i}(\mathcal{O})$ (we say in this case that $I$ is applicable to $S(\boldsymbol{a}, \boldsymbol{b}))$. Then chase $_{i+1}(\mathcal{O})=$ chase $_{i}(\mathcal{O}) \cup\{\psi(\boldsymbol{a}, n)\}$, where $n$ is a fresh constant, i.e., a constant in $\Sigma_{C}$ not occurring in $\operatorname{chase}_{i}(\mathcal{O})$, if $\boldsymbol{z}$ is a single variable in (1), or chase $_{i+1}(\mathcal{O})=$ chase $_{i}(\mathcal{O}) \cup\{\psi(\boldsymbol{a})\}$, if $\boldsymbol{z}$ is absent in (11). We say that chase $_{i+1}(\mathcal{O})$ is obtained from chase $_{i}(\mathcal{O})$ via application of the positive inclusion $I$ to $S(\boldsymbol{a}, \boldsymbol{b})$.

We assume that the chase rule is always executed in such a way that if a positive inclusion $I$ becomes applicable to an ABox assertion $\beta$ in a certain $\operatorname{chase}_{i}(\mathcal{O})$, then there exists $j>i$ such that $\operatorname{chase}_{j}(\mathcal{O})$ is obtained from $\operatorname{chase}_{j-1}(\mathcal{O})$ via application of I to $\alpha$. Then, we call chase of $\mathcal{O}$, denoted chase $(\mathcal{O})$, the set of ABox assertions obtained as the infinite union of all $\operatorname{chase}_{i}(\mathcal{O})$, i.e., $\operatorname{chase}(\mathcal{O})=$ $\bigcup_{i \in \mathbb{N}}$ chase $_{i}(\mathcal{O})$. Associated to the chase, we consider the so-called canonical interpretation of $\mathcal{O}$, denoted can $(\mathcal{O})$, in which every constant is interpreted by itself, and for every predicate $S$, we have that $S^{\operatorname{can}(\mathcal{O})}=\{\boldsymbol{a} \mid S(\boldsymbol{a}) \in \operatorname{chase}(\mathcal{O})\}$. It is possible to show that $\operatorname{can}(\mathcal{O})$ is a model of $\mathcal{O}[6]$.

Let us now turn back to our proof, and show that from the fact that an arc $\left(A_{1}, A_{2}\right) \notin \alpha\left(\mathcal{E}^{*}\right)$, where $A_{1}$ and $A_{2}$ are atomic concepts, it follows that there does not exist a set $\mathcal{P}$ of positive inclusions in $\mathcal{T}$ such that $\mathcal{P} \models A_{1} \sqsubseteq A_{2}$. The cases of arcs between nodes corresponding to roles or attributes can be proved analogously. Let us consider any set $\mathcal{P} \subseteq \mathcal{T}$ of positive inclusions. To prove the thesis we construct a model $I$ of $\mathcal{P}$ and show that if $\left(A_{1}, A_{2}\right) \notin \alpha\left(\mathcal{E}^{*}\right), I$ is not a model of $A_{1} \sqsubseteq A_{2}$. To this aim, we consider the ABox $\mathcal{A}_{A_{1}}=\left\{A_{1}(d)\right\}$, where $d$ is a constant in $\Sigma_{C}$, and the canonical interpretation $\operatorname{can}\left(\mathcal{O}_{P}\right)$ of the ontology $\mathcal{O}_{P}=\left\langle\mathcal{P}, \mathcal{A}_{A_{1}}\right\rangle$, i.e., the model associated to $\operatorname{chase}\left(\mathcal{O}_{P}\right)$. Since $\operatorname{can}\left(\mathcal{O}_{P}\right)$ is a model of $\mathcal{O}_{P}$, it is also a model of $\mathcal{P}$. We show now that $\operatorname{can}\left(\mathcal{O}_{P}\right)$ is not a model of $A_{1} \sqsubseteq A_{2}$. Let us denote with chase $_{i}\left(\mathcal{O}_{P}\right)$ the chase obtained after $i$ applications of the chase rule. We can now show that $\operatorname{chase}_{i}\left(\mathcal{O}_{P}\right)$ contains an ABox assertion of the form $A(d)$ (resp. $P(d, n), P(n, d)$, or $U(d, n)$ ) if and only if there exists an arc from $A_{1}$ to $A$ (resp. to $\exists P, \exists P^{-}$, or $\delta(U)$ ) in $\mathcal{G}_{\mathcal{P}}^{*}$. The if direction of this property can be easily verified. For the only if direction we proceed by induction on the construction of the chase. The base step is indeed trivial. As for the inductive step, various cases are possible. We consider here the case in which chase $_{i+1}\left(\mathcal{O}_{P}\right)$ contains the fact $A(d)$ that is generated from chase $_{i}\left(\mathcal{O}_{P}\right)$ by applying the axiom $A^{\prime} \sqsubseteq A$ of $\mathcal{P}$ (in fact its FOL version, according to our definition of chase). This means that chase $e_{i}\left(\mathcal{O}_{P}\right)$ contains the ABox assertion $A^{\prime}(d)$, and, by the inductive hypothesis, $\mathcal{G}_{\mathcal{P}}^{*}$ contains the arc $\left(A_{1}, A^{\prime}\right)$. It is easy then to see that $\mathcal{G}_{\mathcal{P}}^{*}$ contains the arc $\left(A_{1}, A\right)$. Other possible cases can be proved in an analogous way. It is now very easy to conclude that $\operatorname{can}\left(\mathcal{O}_{P}\right)$ is not a model of $A_{1} \sqsubseteq A_{2}$, since the arc $\left(A_{1}, A_{2}\right)$ is not in $\alpha\left(\mathcal{E}^{*}\right)$.

We can then easily construct an algorithm, called Compute $\Phi$, that, taken as input an OWL 2 QL TBox $\mathcal{T}$, first builds the digraph $\mathcal{G}_{\mathcal{T}}=(\mathcal{N}, \mathcal{E})$ according 
to Definition 2, then computes its transitive closure, and finally returns the set $\Phi_{\mathcal{T}}$, which contains an inclusion assertion $S_{1} \sqsubseteq S_{2}$ for each $\operatorname{arc}\left(S_{1}, S_{2}\right) \in \alpha\left(\mathcal{E}^{*}\right)$.

According to Theorem 2. Compute $\Phi$ is sound and complete with respect to the problem of computing $\Phi_{\mathcal{T}}$ for any OWL 2 QL TBox $\mathcal{T}$ containing only positive inclusions.

Computation of $\Omega_{\mathcal{T}}$. In OWL 2 QL, unsatisfiability of concepts, roles, and attributes can mainly arise due to a malicious interaction of negative and positive inclusions. However, also disjoint value-domains, i.e., datatypes having empty intersection of their value spaces, can cause unsatisfiability. This can happen, due to the presence in the TBox of ill-defined value-domain inclusions, which can make one derive contradictory information. For instance, consider the TBox $\mathcal{T}$ containing the assertions $\rho(U) \sqsubseteq$ xsd:dateTime and $\rho(U) \sqsubseteq$ xsd:integer. Since the xsd:dateTime and xsd:integer datatypes are disjoint, we have that $\mathcal{T} \models U \sqsubseteq \neg U$. The detection of the situation above described is rather technical, and does not add particular value to our overall technique for identification of unsatisfiable predicates. Furthermore, this situation is quite rare in the practice (for example, no ill-typed attributes are present in the benchmark ontologies used in Section (4). Therefore, for the sake of presentation, we prefer here to not consider this case, and assume that the TBox does not contain value-domain inclusions. Furthermore, since under such assumption the treatment of attributes and roles is analogous, we limit here our attention to the case where the TBox does not contain axioms involving attributes. All results given below apply however also to full-fledged OWL 2 QL TBoxes.

We first observe that, according Definition 2 , no node corresponding to a qualified existential role is created in the TBox digraph representation. This kind of node is indeed not useful for computing $\Phi_{\mathcal{T}}$. Differently, if one aims to identify every cause of unsatisfiability, the creation of nodes corresponding to a qualified existential role is needed. This is due to the fact that a TBox may entail that a qualified existential role $\exists P . A$ is unsatisfiable, even in case of satisfiability of $\exists P$. Specifically, this may occur in two instances: $(i)$ if the TBox $\mathcal{T}$ entails the assertion $\exists P^{-} \sqsubseteq \neg A$, and (ii), the TBox $\mathcal{T}$ entails $A \sqsubseteq \neg A$. Clearly, in both cases the concept $\exists P$. $A$ is unsatisfiable. We therefore modify here Definition 2 by substituting Rule 7 with the following one:

$7^{*}$. for each concept inclusion $B_{1} \sqsubseteq \exists Q . A \in \mathcal{T}, \mathcal{N}$ contains the node $\exists Q . A$, and $\mathcal{E}$ contains the arches $\left(B_{1}, \exists Q . A\right)$ and $(\exists Q . A, \exists Q)$;

From now on, we adopt the digraph representation built according to Definition 2. where rule $7^{*}$ replaces rule 7 , and, according to the above assumptions, we consider only OWL 2 QL TBoxes that do not contain axioms involving attributes in $\Sigma_{P}$. Given one such TBox $\mathcal{T}$ over a signature $\Sigma_{P}$, the algorithm computeUnsat given in Figure 1 returns all unsatisfiable concepts and roles in $\Sigma_{P}$, by exploiting the transitive closure of the digraph representation of $\mathcal{T}$.

Before describing the algorithm, we recall that, given a digraph $\mathcal{G}=(\mathcal{N}, \mathcal{E})$ and a node $n \in \mathcal{N}$, the set predecessors $\left(n, \mathcal{G}^{*}\right)$ contains all those nodes $n^{\prime}$ in $\mathcal{N}$ such that $\mathcal{G}^{*}$ contains the $\operatorname{arc}\left(n^{\prime}, n\right)$, which means that there exists a path from $n^{\prime}$ 


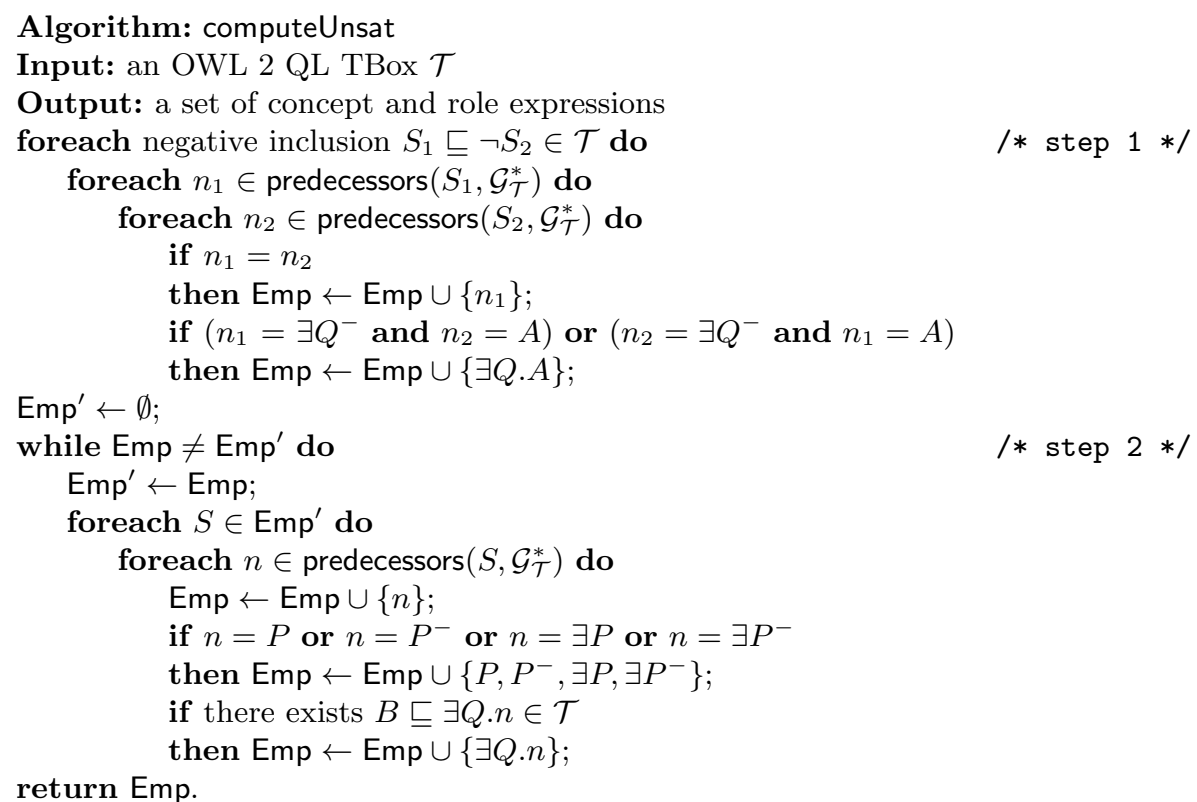

Fig. 1. The algorithm computeUnsat $(\mathcal{T})$

to $n$ in $\mathcal{G}$. Also, it can be shown that $\mathcal{G}_{\mathcal{T}}^{*}$ allows in fact to obtain all subsumptions between satisfiable basic concepts or roles, in the sense that the TBox $\mathcal{T}$ infers one such subsumption $S_{1} \sqsubseteq S_{2}$ if and only if there is an $\operatorname{arc}\left(S_{1}, S_{2}\right)$ in $\mathcal{E}^{*}$. Then, the two steps that compose the algorithm proceed as follows:

Step 1. Let $S$ be either a concept expression or a role expression. We have that for each $S^{i} \in \operatorname{predecessors}\left(S, \mathcal{G}_{\mathcal{T}}^{*}\right)$ the $\operatorname{TBox} \mathcal{T}$ entails $S^{i} \sqsubseteq S$. Hence, given a negative inclusion assertion $S_{1} \sqsubseteq \neg S_{2}$, for each $S_{1}^{i} \in$ $\operatorname{predecessors}\left(S_{1}, \mathcal{G}_{\mathcal{T}}^{*}\right)$ and for each $S_{2}^{j} \in \operatorname{predecessors}\left(S_{2}, \mathcal{G}_{\mathcal{T}}^{*}\right), \mathcal{T} \models S_{1}^{i} \sqsubseteq$ $\neg S_{2}^{j}$. Therefore, for each negative inclusion $S_{1} \sqsubseteq \neg S_{2} \in \mathcal{T}$, the algorithm computes the set predecessors $\left(S_{1}, \mathcal{G}_{\mathcal{T}}^{*}\right)$ and predecessors $\left(S_{2}, \mathcal{G}_{\mathcal{T}}^{*}\right)$ and is able to: $(i)$ recognize as unsatisfiable all those concepts and roles whose corresponding nodes occur in both the set predecessors $\left(S_{1}, \mathcal{G}_{\mathcal{T}}^{*}\right)$ and predecessors $\left(S_{2}, \mathcal{G}_{\mathcal{T}}^{*}\right)$, and $(i i)$ identify those unsatisfiable qualified existential roles $\exists Q$.A whose inverse existential role node $\exists Q^{-}$occurs in $\operatorname{predecessors}\left(S_{1}, \mathcal{G}_{\mathcal{T}}^{*}\right)$ (resp. $\operatorname{predecessors}\left(S_{2}, \mathcal{G}_{\mathcal{T}}^{*}\right)$ ) and whose concept node $A$ occurs in predecessors $\left(S_{2}, \mathcal{G}_{\mathcal{T}}^{*}\right)$ (resp. predecessors $\left(S_{1}, \mathcal{G}_{\mathcal{T}}^{*}\right)$ ), which indeed implies $\exists Q^{-} \sqsubseteq \neg A$ and therefore unsatisfiability of $\exists Q$. $A$.

Step 2. Further unsatisfiable concepts and roles are identified by the algorithm through a cycle in which: $(i)$ if a concept or role $S$ is in Emp, then all the expressions corresponding to the nodes in predecessors $\left(S, \mathcal{G}_{\mathcal{T}}^{*}\right)$ are in Emp. This captures propagation of unsatisfiability through chains of positive inclusions; (ii) if at least one of the expressions $P, P^{-}, \exists P, \exists P^{-}$is in Emp, then all four expressions are in Emp; (iii) for each expression $\exists Q . A$ in $\mathcal{N}$, if $A \in \mathrm{Emp}$, 
then $\exists Q . A \in$ Emp. We notice that the algorithm stops cycling when no new expressions of the form $\exists Q$ or $\exists Q . A$ are added to Emp (indeed, in this case only a single further iteration may be needed).

It easy to see that, by virtue of the fact that the size of the set $\mathcal{N}$ of the digraph representation of the $\operatorname{TBox} \mathcal{T}$ is finite, computeUnsat $(\mathcal{T})$ terminates, and that the number of executions of the while cycle is less than or equal to $|\mathcal{N}|$.

The following theorem shows that algorithm computeUnsat can be used for computing the set containing all the unsatisfiable concepts and roles in $\mathcal{T}$.

Theorem 3. Let $\mathcal{T}$ be an $O W L 2 Q L$ TBox without axioms involving attributes and let $S$ be either an atomic concept or an atomic role in $\Sigma_{P} . \mathcal{T} \models S \sqsubseteq \neg S$ if and only if $S \in$ computeUnsat $(\mathcal{T})$.

As already said, it is easy to extend computeUnsat in such a way that it returns all unsatisfiable atomic concepts, atomic roles, and attributes occurring in general OWL 2 QL TBoxes. Therefore, we can restate Theorem 3 considering OWL 2 QL ontologies with also attributes and value-domain inclusions, and $S$ that can be also an attribute. As an immediate consequence of this, we can compute the set $\Omega_{\mathcal{T}}$ of all "trivial" inclusion assertions inferred by an OWL 2 QL ontology $\mathcal{T}$, by means of the unsatisfiable predicates identified by computeUnsat. We call Compute $\Omega$ the algorithm that, taken $\mathcal{T}$ as input, returns $\Omega_{\mathcal{T}}$ by making use of computeUnsat.

The following theorem, which is a direct consequence of Theorem 2 and of (the generalized version of) Theorem [3, states that our technique is sound and complete with respect to the problem of classifying an OWL 2 QL TBox.

Theorem 4. Let $\mathcal{T}$ be an $O W L 2 Q L$ TBox and let $S_{1}$ and $S_{2}$ be either two atomic concepts, two atomic roles, or two attributes. $\mathcal{T} \models S_{1} \sqsubseteq S_{2}$ if and only if $S_{1} \sqsubseteq S_{2} \in$ Compute $\Phi(\mathcal{T}) \cup$ Compute $\Omega(\mathcal{T})$.

\section{Implementation and Evaluation}

By exploiting the results presented in Section 3, we have developed a Java-based OWL 2 QL classification module for the QUONTO reasoner [1/5]7.

This module computes the classification of an OWL 2 QL TBox $\mathcal{T}$ by adopting the technique described in Section 3. In this implementation the transitive closure of the digraph $\mathcal{G}_{\mathcal{T}}$ is based on a breadth first search through $\mathcal{G}_{\mathcal{T}}$.

We have performed comparative experiments, where QUONTO was tested against several popular ontology reasoners. Specifically, during our test we compared ourselves with the Fact ++ [23], Hermit [9], and Pellet [22] OWL reasoners, and with the $\mathrm{CB}[14$ Horn $\mathcal{S H \mathcal { I }}$ reasoner, and with the ELK [15] reasoner for those ontologies that are also in OWL 2 EL.

The ontology suite used during testing includes twenty OWL ontologies, assembled from the TONES Ontology Repository 1 and from other independent sources. The six reasoners exhibited negligible differences in performance for the

${ }^{1}$ http://owl.cs.manchester.ac.uk/repository/ 
Table 1. In the table the Original and OWL 2 QL axioms fields indicate respectively the total number of axioms in the original version of the ontology and in the OWL 2 QL-approximated version. The Negative inclusion field reports the number of negative inclusions in the OWL 2 QL-approximated version.

\begin{tabular}{|l|r|r|r|c|r|r|r|}
\hline Ontology & Concepts & Roles & Attributes & $\begin{array}{c}\text { Original DL } \\
\text { fragment }\end{array}$ & $\begin{array}{c}\text { Original } \\
\text { axioms }\end{array}$ & $\begin{array}{c}\text { Owl 2 QL } \\
\text { axioms }\end{array}$ & $\begin{array}{c}\text { Negative } \\
\text { inclusions }\end{array}$ \\
\hline Mouse & 2753 & 1 & 0 & $A L E$ & 3463 & 3463 & 0 \\
Transportation & 445 & 89 & 4 & $A L C H(D)$ & 931 & 931 & 317 \\
DOLCE & 209 & 313 & 4 & $S H O I N(D)$ & 1736 & 1991 & 45 \\
AEO & 760 & 47 & 16 & $S H I N(D)$ & 3449 & 3432 & 1957 \\
Gene & 26225 & 4 & 0 & $S H$ & 42655 & 42655 & 3 \\
EL-Galen & 23136 & 950 & 0 & $E L H$ & 46457 & 48026 & 0 \\
Galen & 23141 & 950 & 0 & $A L E H I F+$ & 47407 & 49926 & 0 \\
FMA 1.4 & 6488 & 165 & 0 & $A L C O I F$ & 18612 & 18663 & 0 \\
FMA 2.0 & 41648 & 148 & 20 & $A L C O I F(D)$ & 123610 & 118181 & 0 \\
FMA 3.2.1 & 84454 & 132 & 67 & $A L C O I F(D)$ & 88204 & 84987 & 0 \\
FMA-OBO & 75139 & 2 & 0 & $A L E$ & 119558 & 119558 & 0 \\
\hline
\end{tabular}

majority of the smaller tested ontologies, so we will only discuss the ontologies which offered interesting results, meaning those on which reasoning times are significantly different for at least a subset of the reasoners.

These ontologies include: the Mouse ontology; the Transportation ontology 2; the Descriptive Ontology for Linguistic and Cognitive Engineering (DOLCE) [18; the Athletic Events Ontology (AEO) 3; the Gene Ontology (GO) 2]; two versions of the GALEN ontology [21]; and four versions of the Foundational Model of Anatomy Ontology (FMA) [10.

Because QUONTO is an OWL 2 QL reasoner, each benchmark ontology was preprocessed prior to classification in order to fit OWL 2 QL expressivity. Therefore, every OWL expression which cannot be expressed by OWL 2 QL axioms was approximated from the ontology specifications. This approximation follows this procedure: each axiom in the ontology is fed to an external reasoner, specifically Hermit, and every OWL 2 QL-compliant axiom that is implied from that axiom, between the ontology symbols that appear in it, is added to the OWL 2 QL-approximated ontology. For instance, the OWL assertion EquivalentClasses(ObjectUnionOf(:Male :Female) :Person) is approximated by the two assertions SubClassOf(:Male :Person) and SubClassOf(:Female :Person). Note that, as is the case in this example, the OWL 2 QL-approximated ontology may contain a greater number of axioms than the original ontology.

During the tests for each reasoner, classification was performed on the OWL 2 QL-compliant versions of the ontologies resulting from the above described preprocessing. Metrics about the ontologies are reported in Table 1 .

All tests were performed on a DELL Latitude E6320 notebook with Intel Core i7-2640M 2.8Ghz CPU and 4GB of RAM, running Microsoft Windows 7 Premium operating system, and Java 1.6 with 2GB of heap space. Classification timeout was set at one hour, and aborting if maximum available memory was

\footnotetext{
2 http://www.daml.org/ontologies/409

3 http://www.boemie.org/deliverable_d_3_5
} 
Table 2. Classification times of benchmark OWL 2 QL ontologies by QuONTO and other tested reasoners

\begin{tabular}{|c|c|c|c|c|c|c|}
\hline Ontology & QUONTO & $\mathrm{FaCT}++$ & HermiT & Pellet & $\mathrm{CB}$ & ELK \\
\hline Mouse & 0.156 & 0.282 & 0.296 & 0.179 & 0.159 & 0.246 \\
\hline Transportation & 0.150 & 0.045 & 0.163 & 0.151 & 0.195 & 0.343 \\
\hline DOLCE & 1.327 & 0.245 & 25.619 & 1.696 & 1.358 & - \\
\hline $\mathrm{AEO}$ & 0.650 & 0.743 & 0.920 & 0.647 & 0.605 & - \\
\hline Gene & 1.255 & 1.400 & 3.810 & 2.803 & 1.918 & 1.419 \\
\hline EL-Galen & 2.788 & 109.835 & 7.966 & 50.770 & 2.446 & 1.205 \\
\hline Galen & 4.600 & 145.485 & 34.608 & timeout & 2.505 & - \\
\hline FMA 1.4 & 0.688 & timeout & 93.781 & timeout & 1.243 & - \\
\hline FMA 2.0 & 4.111 & out of memory & out of memory & timeout & 7.142 & - \\
\hline FMA 3.2.1 & 4.146 & 4.576 & 11.518 & 24.117 & 4.976 & - \\
\hline FMA-OBO & 4.827 & timeout & 50.842 & 16.852 & 7.433 & 4.078 \\
\hline
\end{tabular}

exhausted. All figures reported in Table 2 are in seconds, and, because classification results are subject to minor fluctuation, particularly when dealing with large ontologies, are the average of 3 classifications of the respective ontologies with each reasoner. The following versions of the OWL reasoners were tested: Fact ++ v.1.5.34, HermiT v.1.3.65, Pellet v.2.3.66, CB v.127, and ELK v.0.3.28.

In our test configuration, the classifications of the FMA 2.0 ontology by the Hermit and $\mathrm{FaCT}++$ reasoners terminate due to an out-of-memory error. In [9], classification of this ontology by the Hermit reasoner is performed successfully, but classification time far exceeds the one registered by QUONTO.

The results of the experiments are summarized in Table 2 . These results confirm that the performance offered by QUONTO compares favorably to other reasoners for almost all tested ontologies. Classification for even the largest of the tested ontologies, i.e., the FMA-OBO and FMA 3.2.1 ontologies, is performed in under 5 seconds, and memory space issues were never experienced during our tests with QUONTO. For some test cases, the gap in performance between QuONTO and other reasoners is sizeable: for instance, classification by Pellet of the Galen and FMA (1.4 and 2.0) and by FaCT++ of the FMA (1.4 and OBO) ontologies exceeds the predetermined timeout limit of one hour.

Detailed analysis of the results provided in Table 2 shows that only the CB and ELK reasoners consistently display comparable performances to QUONTO, which is fastest for all ontologies which feature only positive inclusions, with the exception of the EL-Galen, Galen, and FMA-OBO ontologies. The CB reasoner, which is the best-performing reasoner for the Galen ontology, does not however always perform complete classification. For instance, it does not compute property hierarchies. The ELK reasoner instead is slower than QUONTO for three out of the five ontologies also in OWL 2 EL, showing instead markedly better performance for EL-Galen.

\footnotetext{
4 http://code.google.com/p/factplusplus/

5 http://hermit-reasoner.com/

6 http://clarkparsia.com/pellet

7 http://code.google.com/p/cb-reasoner/

8 http://code.google.com/p/elk-reasoner/
} 
Furthermore, if, as it is usually the case, an ontology does not present unsatisfiable predicates, the computation of such predicates through the exploration of all negative inclusions can be avoided. This is the case for ontologies such as DOLCE and AEO, for which computation of the set $\Phi_{\mathcal{T}}$ of positive inclusion assertions resulting from the transitive closure of $\mathcal{G}_{\mathcal{T}}$ is performed respectively in 0.347 and 0.384 seconds, fastest among tested reasoners. Instead, for ontologies such as Pizza and Transportation, which feature respectively 2 and 62 unsatisfiable atomic concepts, the identification of all such predicates is unavoidable, and the resulting set of trivial inclusion assertions must be added to $\Omega_{\mathcal{T}}$.

\section{Conclusions}

The research presented in this paper can be extended in various directions. First of all, in the implementation of our technique we have adopted a naive algorithm for computing the digraph transitive closure. We are currently experimenting more sophisticated and efficient techniques for this task. We are also working to optimize the procedure through which we identify unsatisfiable predicates. Finally, we are working to extend our technique to compute all inclusions that are inferred by the TBox (which, in OWL 2 QL, are a finite number). In this respect, we notice that through $\mathcal{G}_{\mathcal{T}}^{*}$ it is already possible to obtain the classification of all basic concepts, basic roles, and attributes, and not only that of predicates in the signature, and that, with slight modifications of computeUnsat, we can actually obtain the set of all negative inclusions inferred by an OWL 2 QL TBox. The remaining challenge is to devise an efficient mechanism to obtain all inferred positive inclusions involving qualified existential roles and attribute domains.

Acknowledgments. This research has been partially supported by the EU under FP7 project Optique - Scalable End-user Access to Big Data (grant n. FP7-318338).

\section{References}

1. Acciarri, A., Calvanese, D., De Giacomo, G., Lembo, D., Lenzerini, M., Palmieri, M., Rosati, R.: QuOnto: Querying Ontologies. In: Veloso, M., Kambhampati, S. (eds.) Proc. of AAAI 2005, pp. 1670-1671. AAAI Press/The MIT Press (2005)

2. Ashburner, M., Ball, C., Blake, J., Botstein, D., Butler, H., Cherry, J., Davis, A., Dolinski, K., Dwight, S., Eppig, J., et al.: Gene Ontology: tool for the unification of biology. Nature Genetics 25(1), 25 (2000)

3. Baader, F., Calvanese, D., McGuinness, D., Nardi, D., Patel-Schneider, P.F. (eds.): The Description Logic Handbook: Theory, Implementation and Applications, 2nd edn. Cambridge University Press (2007)

4. Bang-Jensen, J., Gutin, G.Z.: Digraphs: Theory, Algorithms and Applications, 2nd edn. Springer (2008)

5. Calvanese, D., De Giacomo, G., Lembo, D., Lenzerini, M., Poggi, A., RodriguezMuro, M., Rosati, R., Ruzzi, M., Savo, D.F.: The MASTRO system for ontologybased data access. Semantic Web J. 2(1), 43-53 (2011) 
6. Calvanese, D., De Giacomo, G., Lembo, D., Lenzerini, M., Rosati, R.: Tractable reasoning and efficient query answering in description logics: The DL-Lite family. J. of Automated Reasoning 39(3), 385-429 (2007)

7. De Giacomo, G., Lembo, D., Lenzerini, M., Poggi, A., Rosati, R., Ruzzi, M., Savo, D.F.: MASTRO: A reasoner for effective ontology-based data access. In: Proc. of ORE 2012. CEUR, vol. 858 (2012), ceur-ws.org

8. Fagin, R., Kolaitis, P.G., Miller, R.J., Popa, L.: Data exchange: Semantics and query answering. In: Calvanese, D., Lenzerini, M., Motwani, R. (eds.) ICDT 2003. LNCS, vol. 2572, pp. 207-224. Springer, Heidelberg (2002)

9. Glimm, B., Horrocks, I., Motik, B., Shearer, R., Stoilos, G.: A novel approach to ontology classification. J. of Web Semantics 14, 84-101 (2012)

10. Golbreich, C., Zhang, S., Bodenreider, O.: The foundational model of anatomy in OWL: Experience and perspectives. J. of Web Semantics 4(3), 181-195 (2006)

11. Haarslev, V., Möller, R.: RACER system description. In: Goré, R., Leitsch, A., Nipkow, T. (eds.) IJCAR 2001. LNCS (LNAI), vol. 2083, pp. 701-706. Springer, Heidelberg (2001)

12. Ji, Q., Haase, P., Qi, G., Hitzler, P., Stadtmüller, S.: RaDON — repair and diagnosis in ontology networks. In: Aroyo, L., et al. (eds.) ESWC 2009. LNCS, vol. 5554, pp. 863-867. Springer, Heidelberg (2009)

13. Kalyanpur, A., Parsia, B., Sirin, E., Hendler, J.A.: Debugging unsatisfiable classes in OWL ontologies. J. of Web Semantics 3(4), 268-293 (2005)

14. Kazakov, Y.: Consequence-driven reasoning for Horn $\mathcal{S H \mathcal { L }}$ ontologies. In: Boutilier, C. (ed.) Proc. of IJCAI 2009, pp. 2040-2045. AAAI Press (2009)

15. Kazakov, Y., Krötzsch, M., Simančík, F.: Concurrent classification of $\mathcal{E} \mathcal{L}$ ontologies. In: Aroyo, L., Welty, C., Alani, H., Taylor, J., Bernstein, A., Kagal, L., Noy, N., Blomqvist, E. (eds.) ISWC 2011, Part I. LNCS, vol. 7031, pp. 305-320. Springer, Heidelberg (2011)

16. Krötzsch, M.: The not-so-easy task of computing class subsumptions in OWL RL. In: Cudré-Mauroux, P., et al. (eds.) ISWC 2012, Part I. LNCS, vol. 7649, pp. 279-294. Springer, Heidelberg (2012)

17. Lawley, M., Bousquet, C.: Fast classification in Protégé: Snorocket as an OWL 2 EL reasoner. In: Meyer, T., Orgun, M., Taylor, K. (eds.) Proc. of AOW 2010. CRPIT, vol. 122 , pp. 45-50. ACS (2010)

18. Masolo, C., Borgo, S., Gangemi, A., Guarino, N., Oltramari, A., Schneider, L.: The wonderweb library of foundational ontologies and the DOLCE ontology. Technical Report D17, WonderWeb (2002)

19. Mendez, J., Ecke, A., Turhan, A.: Implementing completion-based inferences for the $\mathcal{E} \mathcal{L}$-family. In: Proc. of DL 2011. CEUR, vol. 745 (2011), ceur-ws.org

20. Motik, B., Grau, B.C., Horrocks, I., Wu, Z., Fokoue, A., Lutz, C.: OWL 2 Web Ontology Language - Profiles, 2nd edn. W3C Recommendation, World Wide Web Consortium (December 2012), http://www.w3.org/TR/ow12-profiles/

21. Rogers, J., Rector, A.: The GALEN ontology. In: Medical Informatics Europe, MIE 1996, pp. 174-178 (1996)

22. Sirin, E., Parsia, B., Grau, B.C., Kalyanpur, A., Katz, Y.: Pellet: A practical OWLDL reasoner. J. of Web Semantics 5(2), 51-53 (2007)

23. Tsarkov, D., Horrocks, I.: FaCT++ description logic reasoner: System description. In: Furbach, U., Shankar, N. (eds.) IJCAR 2006. LNCS (LNAI), vol. 4130, pp. 292-297. Springer, Heidelberg (2006) 